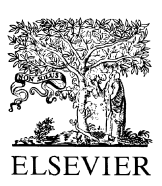

\title{
Sequential Liver Transplantation: 27 Cases in 25 Patients
}

\author{
L. Tomé, J. Ferrão, E. Furtado, J. Geraldes, O. Mota, F. Oliveira, R. Perdigoto, J. Viana, A. Cipriano, \\ and L. Furtado
}

$\mathrm{F}^{\mathrm{s}}$ AMILIAL amyloid polyneuropathy (FAP) is the most common form of herederitary amyloidosis. Initial clinical manifestation is a sensoriomotor polyneuropathy starting in the lower limbs and later affecting the upper extremities. The autonomic nervous system is involved (orthostatic hypotension and impotence). Severe constipation (often the first gastrointestinal symptom) is relieved with time by bouts of diarrhea that subsequently become continuous. Cardiac arrhytmias are common. Some patients develop renal failure because of accumulation of amyloid in the kidneys together with bladder denervation. ${ }^{1}$

FAP is characterized by systemic deposition of amyloid. Amyloid fibrils are composed of variant transthyretin in which a single amino acid substitution (TTRMet30 in FAP type 1) results in the aggregation and insolubility of the protein with deposition in extracellular spaces. More than $95 \%$ of TTR is produced in the liver. ${ }^{2}$ Apart from the production of the variant transthyretin, FAP livers are functionally and anatomically normal. The course of the disease is progressive leading to death within 14 years of the onset of symptoms. Liver transplantation is the only effective therapy. ${ }^{3}$

FAP presents from the third to sixth decade, which suggests that it can take this long for sufficient amyloid to be deposited in the tissues. Elderly asymptomatic TTRMet30 homozygotes have been reported. ${ }^{4}$ Among the heterozygotes, penetrance and rate of disease progression vary substantially. ${ }^{5}$

Portugal has several hundreds of FAP type 1 patients for whom the sole hope of stopping the progression of the disease is liver transplantation. The severe shortage of cadaveric donor organs led us ${ }^{6}$ to consider the use of the liver removed from these subjects in patients awaiting liver transplantation. The lean body habitus and the absence of portal hypertension anticipate an easy hepatectomy. A young stable donor and the possible short cold ischemic time should allow excellent conditions for the recipient. The persistent synthesis of the anomalous TTR by the donated organ creates the potential for development of neuropathy.

\section{METHODS}

From October 1992 to December 31, 1999, 93 cases of FAP were transplanted at Coimbra University Hospital. Twenty-three patients donated the liver, and four livers removed at other Institutions were accepted for such an operation. In the same time interval, we transplanted 110 patients older than 12 , not affected by FAP, and not involved in sequential liver transplantation.

The livers removed from FAP patients were offered to recipients in desperate condition older than 50. Later on, younger patients were also considered as potential recipients if the success of the transplantation could be jeopardized by their clinical condition. In recent times, any patient with cirrhosis older than 50 was asked permission for sequential liver transplantation. In all, 25 patients acted as recipients in the sequential procedure.

Facing an appropriate clinical picture the diagnosis of type 1 FAP was formulated by identification of circulating TTRMet 30 . The neurologic deficit of these patients was quantified. ${ }^{7}$ These patients were extensively studied before the transplant to exclude the presence of liver disease other than the metabolic disturbance. When indicated, the native hepatectomy in the FAP patient implied technical specificities necessary for the donation. The suprahepatic inferior vena cava was dissected into the intrapericardial portion with division of the phrenic veins and division of the diaphragmatic fibrotic ring to ensure sufficient length for both the implantation of the cadaveric graft and use of the liver as a domino graft. The hepatic artery was divided at the level of the gastroduodenal artery and the portal vein was divided within $1 \mathrm{~cm}$ of the bifurcation.

Both FAP donors and recipients gave informed written consent after receiving extensive counseling and information about technical aspects of the surgery and the potential risk to the recipient of developing FAP. The possibility of retransplantation was offered, should FAP develop in the absence of tumor recurrence. The Ethics Committee at Coimbra University Hospital approved the procedure.

\section{RESULTS}

The 27 FAP patients that donated their livers were $35.6 \pm$ 7.2 years old (range 26 to 54 ). The neurologic disability scored $30.7 \pm 11.7 \%$ (range 8 to 65 ). The symptoms caused $4.4 \pm 1.6$ years before transplantation (range 1 to 7 ). The BMI was $21.8 \pm 3.9 \mathrm{~kg} / \mathrm{m}^{2}$ (range 14.7 to 29.5 ).

The cold ischemic time (CIT) was $588.4 \pm 156.7$ minutes (range 320 to 913 minutes, CIT $>720$ minutes in 5 patients or $23.8 \%$ ). Arterial hepatic thrombosis was detected in one case. A biliary leak presented in another patient. Other major complications were acute renal insufficiency and

From the Transplantation Department, University Hospital, Coimbra, Portugal.

Address reprint requests to $\mathrm{Dr}$ L. Tome, Hospital da Universidate, Department de Transplantacoes, 3000 Coimbra, Portugal. 
splenic flexure necrosis. Four out the 23 donors operated in our hospital were retransplanted for PNF, $(n=2)$; hepatic artery thrombosis, or de novo hepatitis C (retransplanted at 14 months).

Three patients died (13\%). One patient died after de novo $\mathrm{C}$ virus infection and retransplantation. Another died suddenly at home 18 months after transplantation (type 2 Mobitz AV block was known). One patient died after a sudden cardiac arrest at the end of transplantation (a pacemaker had been introduced immediately before the operation). Actuarial survival for this cohort was $95.6 \%$ at 30 days, $95.4 \%$ at 6 months, and $95.0 \%$ at 1 year.

CIT for the 70 FAP patients (similar age, neurologic, score and BMI) not involved in the sequential procedure was $495 \pm 141.1 .7$ minutes (CIT $>720$ minutes, $3.0 \%$ ). Hepatic artery thrombosis was detected in $5.7 \%$, PNF in $1.4 \%$. Eleven percent were retransplanted. Actuarial survival was $100 \%$ at day $30,89.5 \%$ at 6 months, and $81.9 \%$ at 1 year (significantly only for CIT $>720$ minutes).

Twenty-five patients received 27 livers removed from FAP patients. Liver cirrhosis was known in 16 cases, and metastatic disease in two cases. Other diagnosis were present in 7 cases (hemangioendothelioma in two cases, relapse of hepatocellular carcinoma, hepatocholangiocarcinoma, acute Budd-Chiari syndrome, acute hepatic failure, hepatocellular carcinoma in normal liver). Hepatocellular carcinoma was suspected or known in 11 cases (actually present in 9).

These patients were $48.8 \pm 9.1$ years old (range 28 to 60 ). We transplanted four cases younger than 40 (28 years old, Budd-Chiari, third transplant; 29 years old, hemangioendothelioma; 33 years old, hepatocholangiocarcinoma; 34 years old, acute hepatic failure, third transplant). Seven more cases were younger than 50 (40 years old, HBV + HVC + hepatocellular carcinoma + hepatopulmonary syndrome; 44 years old, hepatocellular carcinoma in normal liver; 44 years old, acute hepatic failure; 44 years old, HBV + hepatocellular carcinoma; 44 years old, alcoholic cirrhosis; 47 years old, Wilson disease, hepatocellular carcinoma; and 48 years old, neuroendocrine metastasis).

CIT was $457 \pm 198$ minutes (range 135 to 795 minutes). CIT was less than 300 minutes in seven cases $(25.9 \%)$. CIT was over 720 minutes in four patients (14.8\%). Arterial hepatic thrombosis was detected in three cases (15.7\%). Biliary stenosis developed in two cases. A biliary leak was detected in one patient. The sequential procedure was the first transplant in 20 cases. Two patients were retransplanted.

Twelve patients died. Death was ascribed to infections in five patients, tumoral relapse in four cases, renal failure, cererovascular accident, and multiorgan failure. Actuarial survival for this cohort was $88.0 \%$ at day $30,62.5 \%$ at 6 months, and $50.0 \%$ at 1 year.

For comparison, in the 110 patients older than 12, not affected by FAP, and not involved in sequential liver transplantation operated in the same time interval in our center, the retransplantation rate was $11.8 \%(P=\mathrm{NS})$. The actuarial survival of these group was $84.3 \%$ at day $30,74.4 \%$ at 6 months, and $72.1 \%$ at 1 year (significant difference at 1 year).

The recipients were strictly followed to ascertain the emergence of a polyneuropathy. One case was transplanted more than 5 years ago, after being diagnosed an hepatocellular carcinoma superimposed on a $\mathrm{C}$ cirrhosis. No tumoral recurrence occurred. He has no neurologic symptoms. Electromyography of lower limbs is normal. A skin biopsy (17.02.99) was negative for amyloid.

\section{CONCLUSIONS}

With the remarkable exception of producing an anomalous protein, the liver in patients afflicted by FAP functions well. With this premise in mind, our group introduced sequential liver transplantation in 95 patients whereby the liver removed from a patient suffering from FAP is transferred to another patient. In the meantime, the FAP donor receives a cadaveric organ.

Mean CIT for cadaveric livers implanted in donors involved is not different from that observed for livers transplanted in non donors $(588.4 \pm 156.7$ vs $495.2 \pm 141.1$ minutes). Ischemic times greater than 720 minutes are more frequent in donors $(23.8 \%$ vs $3.5 \%)$ but this difference is not to be ascribed to the procedure. We observed an identical prevalence of PNF $(8.7 \%$ vs $1.4 \%)$ or hepatic artery thrombosis $(4.3 \%$ vs $5.7 \%)$ in both groups. The hepatectomy on the donor requires technical specificities not necessary for the normal transplantation. The results of this series clearly demonstrate that the modifications do not increase the number of major complications in the patients that donated their livers, neither increase the frequency of retransplantation $(17.4 \%$ vs $11.4 \%)$. It is also clear that the sequential procedure do not compromise the survival of FAP donors (at 1 year, $76.9 \%$ vs $80.7 \%$ ) or jeopardize the survival of any individual case.

The actuarial survival of the recipients of FAP livers (at 12 months, $54.1 \%$ ) is clearly inferior to that observed by our group for individuals older than 12, without FAP, and not involved in sequential liver transplantation (at 12 months, $72.1 \%$ ). The low actuarial survival of this cohort should be ascribed to the extreme condition of most of the involved cases. Only $8(57.1 \%)$ out of 14 patients harbouring a tumor are currently alive. Only $5(45.4 \%)$ out of 11 patients younger than 50 survived the procedure.

With only one recipient alive more than 5 years after a sequential liver transplantation, it is probably too early to ascertain the emergence of the neuropathy in the receptors. It should be stressed that, in this particular cirrhotic patient, neurologic symptoms are not present, electromyographic studies are normal, and skin biopsies do not reveal amyloid deposition.

\section{REFERENCES}

1. Coelho T: Curr Opin Neurol 9:355, 1996

2. Sariava M, Costa P, Birken S, et al: Trans Assoc Am Phys 96:261, 1983 
3. Suhr O, Holmgren G, et al: Transplantation 60:933, 1995

4. Ikeda S, Nakanamo T, et al: Eur Neurol 32:308, 1992

5. Coelho T, Sousa A, et al: J Med Gen 31:293, 1994
6. Furtado A, Tomé L, Oliveira F, et al: Transplant Proc 29:467, 1997

7. Macedo E, Coutinho P, Morais R, et al: In Isobe T, Araki S, Uchino F, et al (eds): Amyloid and Amyloidosis. New York: Plenum; 1988 